\title{
0617 WHY DO CHILDREN GET BITTEN IN THE FACE?
}

K Meints*, C Syrnyk, T De Keuster Correspondence: University of Lincoln, Brayford Pool LN6 7TS, UK

10.1136/ip.2010.029215.617

The majority of dog bite accidents happen at home and involve children under the age of 7 (Kahn et al, 2003) and a familiar dog. The prevalence of bites in children is double as in the general population (Kahn et al 2004) and often children suffer from dog bites resulting in facial injuries (Bernardo et al, 
2002, Kahn et al, 2003; Schalamon et al, 2006). 55\% children suffer post-traumatic stress disorder following a substantial bite (Peters et al, 2004). According to research these bites are independent of the size of the dog (Kahn et al, 2003), therefore it is unclear why young children get bitten mainly in the face and neck area. As often children's behaviour triggers bite injuries, we studied children's physical distance to an object and the role of smell and mobility of an object on children's facial proximity (leaning in) behaviour. We tested this with novel objects and toy animals in children aged 4, 5 and 6 years. Children showed intrusive facial proximity especially with moving items (eg, animates/ toy animals) or novel items. Children under the age of 5 years showed significantly more proximity behaviours than older children. In addition, we also gathered first evidence that children show clear leaning in behaviour with small animals. Thus, we can now make parents aware of younger children's intrusive inspection behaviour, integrate this knowledge in prevention messages and contribute to reduction in bite injuries, especially to children's faces. 